\title{
Desafio do Núcleo de Atenção Médica Integrada diante da Necessidade de Inserção de Fonoaudiólogo na Rede Municipal de Saúde de Fortaleza
}

\section{The Challenge of Núcleo de Atenção Médica Integrada in View of the Need to Include the Speech-Language Pathologist in Fortaleza's Municipal Health System}

\author{
Maria Emília Macedo Lopes Silva \\ Pedagoga. Fonoaudióloga do Instituto de Otorrino e Cirurgia de \\ Cabeça e Pescoço, Imperatriz, MA, Brasil. \\ Endereço: Rua Alagoas, 6II, Centro, CEP 65919-180, Imperatriz, \\ MA, Brasil. \\ E-mail: emilialpsळhotmail.com \\ Christina Cesar Praça Brasil \\ Fonoaudióloga. Mestre em Distúrbios da Comunicação Humana. \\ Professora da Universidade de Fortaleza. \\ Endereço: Rua Padre Januário Campos, 68, casa 20, Seis Bocas, CEP \\ 60822-840, Fortaleza, CE, Brasil. \\ E-mail: cpracaळunifor.br \\ Aretuza Carla da Fonseca Regis \\ Fonoaudióloga. \\ Endereço: Rua Desembargador João Firmino, 340, Bloco H, ap. 102, \\ CEP 60425-560, Fortaleza, CE, Brasil. \\ E-mail: aretuzafonoळhotmail.com
}

\section{Resumo}

Objetivo: Demonstrar a necessidade da inserção do fonoaudiólogo na rede municipal de saúde de Fortaleza-CE, diante da elevada demanda de usuários e da escassez de serviços gratuitos de fonoaudiologia nesse município. Métodos: Estudo documental, transversal e descritivo com abordagem quantitativa, realizado de julho e setembro de 2008, no qual foram analisados os documentos de atendimentos fonoaudiológicos do Núcleo de Atenção Médica Integrada da Universidade de Fortaleza (NAMI-Unifor), no período de janeiro de 2006 a dezembro de 2007. Resultados: Houve predomínio de atendimento das faixas etárias de o e 6 anos no setor de audiologia, $(22,05 \%)$; e de o a 10 anos no setor de fonoterapia, (58,09\%). 0 exame audiológico mais procurado foi a audiometria (65,78\%); o principal responsável pelo encaminhamento ao setor de audiologia foi o otorrinolaringologista $(56,39 \%)$. No setor de fonoterapia, a linguagem foi a área com maior prevalência de atendimentos $(36,99 \%)$. A terapia em grupo atingiu percentual significativo, com 37,27\%, em 2007. Foi registrada queda no número de atendimentos durante o período de recesso da Universidade, devido à redução do número de alunos. Conclusão: Com um trabalho em nível de atenção primária, no que concerne à saúde da comunicação humana/deglutição, haveria diminuição de gastos públicos com o tratamento das alterações fonoaudiológicas nos demais níveis de atenção. A presença do fonoaudiólogo nas Unidades Básicas de Saúde (UBS) possibilitaria a 
melhoria da qualidade de vida da população em todos os ciclos de vida, além da redução da sobrecarga nos serviços gratuitos que prestam atendimento fonoaudiológico no município de Fortaleza.

Palavras-chave: Comunicação; Promoção da Saúde; Saúde Pública e Coletiva.

\section{Abstract}

Purpose: To demonstrate the need to include the speech-language pathologist and audiologist in the municipal health system of the city of Fortaleza (Northeastern Brazil), due to the high demand of patients and the lack of public speech-language and audiology services in the city. Methods: Documental, cross-sectional and descriptive study with a quantitative approach, developed between July and September 2008. Data were collected through the analysis of the records of the speech-language and audiology assistance provided for patients at NAMIUnifor (The Medical Care Nucleus of the University of Fortaleza) in the period from January 2006 to December 2007. Results: There was predominance of the assistance provided for the age groups between $\mathrm{o}$ and 6 years old in the audiology service (22.05\%) and o to 10 years old in the speech-language therapy service (58.09\%). The most applied audiology procedure was audiometry (65.78\%), and the main professional responsible for referring patients to the audiology service was the otorhinolaryngologist (56.39\%). In speech-language therapy, oral language and/or writing was the area with the highest prevalence of visits (36.99\%). Group therapy reached a significant percentage: $37.27 \%$ in 2007 . A fall in the number of visits during the University's recess period was observed, due to the reduction in the number of students who are responsible for the procedures. Conclusion: With work in the level of primary care as regards the health of human communication/ deglutition, there would be a reduction in public expenses with the treatment of speech-language and audiology alterations in the other health care levels. The presence of the speech-language therapist in the Primary Care Units would help to improve the quality of life of the population across the whole life cycle, and would also reduce the burden in the public services that provide speech-language therapy in the city of Fortaleza.

Keywords: Communication; Health Promotion; Public Health. 


\section{Introdução}

A história do Sistema Único de Saúde (SUS) no Brasil, desde a sua implantação, é repleta de muitas conquistas e inúmeros desafios. Cada experiência bem-sucedida provoca reflexões a ponto de suscitar questionamentos sobre como foi possível esperar tanto tempo por esses progressos.

O SUS foi definido, juntamente com a publicação da Constituição Federal (Brasil, 1988), como um sistema integrado, descentralizado, de ações e serviços que visam à redução do risco de doença e ao acesso universal e igualitário da população a promoção, proteção e recuperação da saúde.

A promoção da saúde deve ser entendida como uma estratégia transversal, multi e interdisciplinar, não se limitando a questões relativas à prevenção, tratamento e cura de doenças (Silva e Araújo, 2007). É necessário, portanto, buscar, na prática, o conceito de saúde instaurado pela Organização Mundial de Saúde desde 1978, segundo o qual saúde é "um estado de completo bem-estar, de equilíbrio biopsicossocial e não somente a ausência de enfermidades" (Befi, 1997, p. 16), ou seja, enxergar o indivíduo como um ser integral, que necessita mais do que a simples "cura” da doença para estar realmente saudável. É necessário ter condições dignas de trabalho, moradia, cidadania e oportunidade aos serviços de saúde que prezem pela promoção em saúde, demonstrando a concretização do princípio da integralidade, preconizado pelo SUS (Befi, 1997; Bleicher, 2004; Marin e col., 2003). Além disso, os serviços de saúde oferecidos devem se mostrar responsáveis pelo cidadão, a ponto de, quando possível, solucionar o problema apresentado e quando não, encaminhá-lo a quem possa solucionar, caracterizando, assim, o princípio da resolutividade (Goulart, 2003).

A partir desse novo conceito, muitas mudanças começam a acontecer e uma estrutura previamente consolidada socialmente passa a ser remodelada para atender às necessidades propostas. Com isso, a formação dos profissionais da área de saúde também sofre modificações, na troca de modelos biomédicos conservadores, pela visão de elevação da qualidade de saúde da população, na organização da gestão setorial e na estruturação do cuidado à saúde, com base no quadrilátero da formação para a área de saúde: ensino, gestão, atenção e controle social (Ceccim e Feuerwerker, 2004, 2006; Brasil, 2004). Nesse contexto, a fonoaudiologia, até então voltada essencialmente à reabilitação, em níveis de atenção secundária e terciária, iniciou a (re)construção de sua identidade e caracterização da práxis em saúde coletiva, buscando definir seu papel junto à promoção da saúde de maneira reflexiva, consciente, responsável e atuante (Penteado e Servilha, 2004). Assim, passou-se a agir em nível de atenção primária, na promoção da saúde no sentido mais amplo e na proteção específica, trabalhando com grupos e/ou com fatores de risco.

Para que a inserção do fonoaudiólogo na rede municipal de saúde de Fortaleza-CE seja realizada, é necessário que os gestores, usuários e outros profissionais conheçam a atuação desse profissional nos três níveis de atenção à saúde, além do seu impacto na prática da integralidade e resolutividade.

Os aspectos envolvidos na habilidade de comunicação do ser humano, como a fala, a voz, a linguagem e a audição, são considerados atributos da saúde. Suas manifestações patológicas comprometem a qualidade de vida (Gonçalves e col., 2005). Com base nisso e em respeito aos princípios do SUS, a Saúde Coletiva foi instaurada como a quinta área de atuação do fonoaudiólogo, considerando que ações preventivas em fonoaudiologia contribuem significativamente para promover a saúde geral em todos os ciclos de vida (Chun, 2004).

A atenção primária constitui o primeiro nível de ação do sistema básico de saúde, devendo estar vinculado de forma integrada e garantir à comunidade a descentralização do serviço de fonoaudiologia e demais serviços públicos de saúde (Paoletti, 1992). Vem sendo observado o aumento da demanda da população por assistência fonoaudiológica. Essa demanda não vem sendo suprida em virtude da inexistência de centros de atendimento fonoaudiológico gratuitos na cidade de Fortaleza-CE.

O fonoaudiólogo pode atuar junto à comunidade, desenvolvendo atividades de promoção e proteção à saúde em geral e da comunicação humana em todos os ciclos de vida. Esse profissional avalia as necessidades locais, trabalhando com observações, avaliações e triagens, orientações e intervenções, nos casos necessários, detectando aspectos que 
possam interferir na comunicação humana (ruído, poluição do ar, falta de vacinação, de pré-natal, presença de maus hábitos orais, respiração oral, possíveis alterações de linguagem, condições de aleitamento materno, de desenvolvimento infantil, saúde auditiva, vocal e possíveis alterações de deglutição) (Basílio e col., 20oo; Pretto e Franco, 1999). Assim, o fonoaudiólogo pode estabelecer parcerias com escolas, creches e entidades representativas da população, a fim de implementar medidas que visam à conscientização da população acerca dos aspectos mencionados.

Considerando a importância da comunicação humana para o desenvolvimento integral do indivíduo, o fonoaudiólogo é um profissional imprescindível na atenção básica à saúde. (Gonçalves e col., 2000; Mendes, 1999). Com base nessa concepção, o Ministério da Saúde regulamentou a inclusão do fonoaudiólogo e de outros profissionais na atenção primária através da criação dos Núcleos de Apoio à Saúde da Família (NASFs) (Brasil, 2008).

O Núcleo de Atenção Médica Integrada NAMI, da Universidade de Fortaleza é um serviço de referência e o maior na área fonoaudiológica no município, que presta atendimento gratuito em níveis secundário e terciário, oferecendo exames complementares à população de Fortaleza e região metropolitana. 0 serviço de fonoaudiologia do NAMI-Unifor, fundado em 1985, funciona como centro de pesquisa e estágio para os alunos dos cursos de graduação e pós-graduação. Possui uma demanda sempre crescente de pacientes, realizando triagens seletiva e eletiva, terapias individual e em grupo, estimulação precoce, grupos de orientação sobre linguagem, motricidade orofacial, voz e audição, ambulatórios de espasticidade e disfagia, e, ainda, todos os procedimentos audiológicos. De 1999 a 2006, o número de atendimentos por ano passou de 24.067 para 34.547. Apesar da ampliação do setor nos últimos anos, observa-se uma procura muito elevada pela assistência fonoaudiológica, o que gera uma demanda freada bastante significativa.

Na cidade de Fortaleza, algumas instituições beneficentes oferecem atendimento fonoaudiológico à população, entretanto, taxas são cobradas por atendimento e isso nem sempre está ao alcance de todos. Poucos hospitais públicos municipais e estaduais dispõem de fonoaudiólogos em suas equipes, os quais não dão vazão ao elevado número de pacientes que lhes são encaminhados, em todas as áreas da Fonoaudiologia. No Estado do Ceará contamos com apenas nove fonoaudiólogos concursados lotados em dois hospitais públicos estaduais. Nos hospitais públicos municipais de Fortaleza, não há fonoaudiólogos contratados, apesar de o cargo existir no quadro funcional do município (Plano de cargos, carreiras e salários da Saúde - Lei nº 7.759 de 24/7/1995) - anexo 1.

Atualmente, encontramos na rede municipal de saúde de Fortaleza 12 fonoaudiólogos contratados em regime de prestação de serviço, os quais dão suporte aos 24 NASFs, compostos de 151 profissionais de oito categorias. Cada núcleo ligado a oito Equipes de Saúde da Família (ESF). Ressalta-se, entretanto, que os fonoaudiólogos lotados nos NASFs ainda são insuficientes para atender à demanda de Fortaleza, uma vez que o município possui atualmente 320 ESFs.

Além disso, o fonoaudiólogo encontra-se timidamente inserido na atenção básica à saúde, porém, os níveis de atenção secundária e terciária encontram-se ainda desassistidos. A maioria das Unidades Básicas de Saúde e as Policlínicas não contam com esse serviço, dificultando os encaminhamentos feitos pelos médicos de diversas especialidades, como também pelos dentistas e professores de ensino fundamental e médio. Essa demanda tem superlotado os poucos serviços disponíveis, gerando na população um nível elevado de insatisfação.

O objetivo deste estudo foi demonstrar a necessidade da inserção do fonoaudiólogo na rede municipal de saúde de Fortaleza-CE, adiante da elevada demanda de usuários e da escassez de serviços gratuitos de fonoaudiologia nesse município, através do levantamento e caracterização do perfil das demandas atendidas e freadas no NAMI-Unifor, de janeiro de 2006 a dezembro de 2007. Para, assim, respaldar a importância desse profissional nos três níveis de atenção à saúde, fundamentando sua atuação na equipe ampliada de saúde da família, de acordo com o princípio da integralidade, além de favorecer a inclusão da fonoaudiologia na ESF, visando à melhoria da atenção básica à saúde. 


\section{Métodos}

Com base nos objetivos expostos, realizou-se uma pesquisa documental, transversal e descritiva, com uma abordagem quantitativa. Foi solicitada a assinatura do Termo de Consentimento Livre e Esclarecido pelo diretor do NAMI-Unifor, conforme resguarda a resolução $n^{0}$ 196/96 do CNS. Este estudo vincula-se ao projeto guarda-chuva "Inserção do Fonoaudiólogo na Rede Municipal de Saúde de Fortaleza: contribuições para a ampliação da integralidade", aprovado pelo Comitê de Ética e Pesquisa da Universidade de Fortaleza, sob parecer $n^{0}$ 131/2008.

A coleta de dados foi realizada entre os meses de julho e setembro de 2008 , durante a qual foram analisados os dados dos documentos de atendimentos fonoaudiológicos, cedidos pela responsável técnica do serviço (prontuários, fichas de triagem, relatórios de avaliação e atendimento fonoaudiológico), além dos relatórios de produtividade, emitidos pelo setor financeiro, presentes no arquivo do setor de fonoaudiologia do NAMI, no período de janeiro de 2006 a dezembro de 2007. Esse foi, portanto, o critério de inclusão, excluindo-se os registros de atendimentos realizados fora desse período.

Para constituir o corpus deste trabalho elaborou-se um formulário que serviu de instrumento de coleta de dados, o qual foi preenchido pelas pesquisadoras durante a análise dos dados, favorecendo a sistematização das informações pertinentes ao estudo, contendo os seguintes itens: diagnóstico situacional (caracterização da instituição quanto ao espaço físico, recursos humanos, rotina da instituição e população atendida); caracterização da população usuária (quanto a gênero, faixa etária, nível de escolaridade, perfil socioeconômico do usuário e origem do encaminhamento); procedimentos realizados e lista de espera dos serviços referente aos múltiplos aspectos da comunicação humana: audição, voz, linguagem e motricidade orofacial. Para a análise e apresentação dos dados, foi utilizada a versão 2007 do programa Excel na construção de gráficos e tabelas, constituindo-se, portanto, a estatística descritiva.

Durante a sistemática da coleta de dados, foram verificados todos os 346 prontuários de atendimentos concluídos (altas, cancelamentos e desistências) no setor de fonoterapia presentes nos arquivos no período estabelecido para a pesquisa. Devido ao extenso número de prontuários do setor de audiologia foi realizado um estudo randomizado, sendo pesquisada uma amostra de 798 prontuários, correspondendo a $29,42 \%$ do total de 2.712 .

\section{Resultados e Discussão}

Objetivando facilitar a compreensão deste estudo, optou-se por separar a apresentação dos dados coletados nos prontuários dos setores de audiologia (798) e fonoterapia (346), considerando que se trata de dinâmicas de atendimento diferenciadas, e, portanto, com preenchimento de documentos distintos para os dois setores.

Os resultados apresentados referem-se ao somatório das informações coletadas nos anos de $2006 \mathrm{e}$ 2007, em virtude de não haver diferenças consideráveis em níveis percentuais nos dois anos e com o objetivo de facilitar a visualização dos dados.

Foram realizados 21.299 procedimentos audiológicos, em um total de 2.712 prontuários, entre janeiro de 2006 e dezembro de 2007 , levando em consideração que um único prontuário traz o registro de diversos procedimentos audiológicos. Desse total, foi analisada uma amostra de 798 prontuários $(29,42 \%)$, nos quais se verificaram aspectos relacionados a gênero, faixa etária, procedimento realizado e origem do encaminhamento.

\section{Perfil da demanda para exames audiológicos}

\section{Perfil dos usuários}

Houve predomínio do gênero feminino, com 59,14\% (472), em relação ao masculino, com 40,85\% (326). Dentre as faixas etárias, obtiveram destaque as crianças entre o e 6 anos, com 22,05\% (176); seguidas de pacientes com idade entre 51 e 65 anos, $15,41 \%$ (123); de 41 a 50 anos, 14,16\% (113); e maiores de 65 anos, $13,65 \%$ (109). As pessoas com idades entre $21 \mathrm{e}$ 30 anos e 31 e 40 anos atingiram o mesmo percentual, $10,40 \%$ (83). As idades de 7 a 10 anos, com 6,14\% (49); 11 a 15 anos, com 4,88\% (39); e 16 a 20 anos, com 2,88\% (23) alcançaram menor prevalência.

Pelo levantamento das faixas etárias percebe-se o efeito do trabalho de sensibilização dos fonoaudiólogos na divulgação da triagem auditiva neonatal e a ampliação do conhecimento da população acerca da avaliação auditiva em crianças, como forma de 
detectar precocemente a perda auditiva.

As pessoas com idade igual ou superior a 41 anos também apresentaram percentual considerável. No Brasil, a redução progressiva da capacidade auditiva devido ao avanço da idade, denominada presbiacusia, vem sendo apontada como causa mais frequente da deficiência auditiva em idosos, com prevalência em torno de 30\% na população com mais de 65 anos de idade (Veras e Matos, 2007).

A presbiacusia é um tipo comum de perda auditiva causada pela degeneração coclear, prejudicando a percepção das frequências altas, interferindo diretamente na compreensão durante a comunicação verbal, consequentemente, nas relações sociais do idoso. Esse fato requer atenção especial dos serviços de saúde, demonstrando a necessidade da presença do fonoaudiólogo nesse contexto. Segundo Portmann e Portmann (1993), esse tipo de perda auditiva é um fenômeno biológico que se inicia, geralmente, a partir de 30 anos de idade, podendo tornar-se socialmente incômodo a partir dos 40 ou 50 anos; o que nos leva a pensar que o relevante número de pessoas encaminhadas ao setor de audiologia do NAMI nessa faixa etária reflete a necessidade da população em conhecer sua condição auditiva, na busca pela melhoria da qualidade de vida.

\section{Procedimentos realizados no setor de audiologia}

Em relação aos procedimentos audiológicos, o cálculo foi baseado no total de atendimentos realizados nos anos de 2006 e 2007, totalizando 21.299 procedimentos, incluindo audiometria, imitanciometria, vectonistagmografia/VENG, pesquisa de potencial auditivo de tronco cerebral/BERA, emissões otoacústicas/EOA, avaliação do processamento auditivo e reabilitação vestibular.

Com o objetivo de facilitar a visualização dos dados, foram considerados, no item "audiometria", todos os tipos de exames audiométricos realizados no NAMI: audiometria tonal limiar adulto e infantil, audiometria vocal com pesquisa do limiar de discriminação, audiometria vocal com pesquisa do limiar de inteligibilidade, audiometria vocal com pesquisa do reconhecimento de fala e audiometria de observação comportamental. Essa medida não compromete a qualidade do estudo, pois, durante a realização das audiometrias, muda-se apenas o método de execução do exame, de acordo com o caso de cada paciente, sendo os objetivos semelhantes.

Houve destaque para a realização dos exames audiométricos, com $65,78 \%$ (14.012), por ser o exame auditivo mais conhecido e de fácil e rápida realização. Em seguida, apareceu a imitanciometria, com 23,36\% (4.976), devendo ser realizada na maioria dos casos de audiometria. A vectonistagmografia apareceu em terceiro lugar, com 3,58\% (764), em seguida, com $3,35 \%$ (715), a pesquisa de potencial auditivo de tronco cerebral (BERA) e emissões otoacústicas (EOA), com 3,54\% (754) (Figura 1).

\section{Figura I - Procedimentos realizados no setor de audiologia do NAMI, 2006-2007}

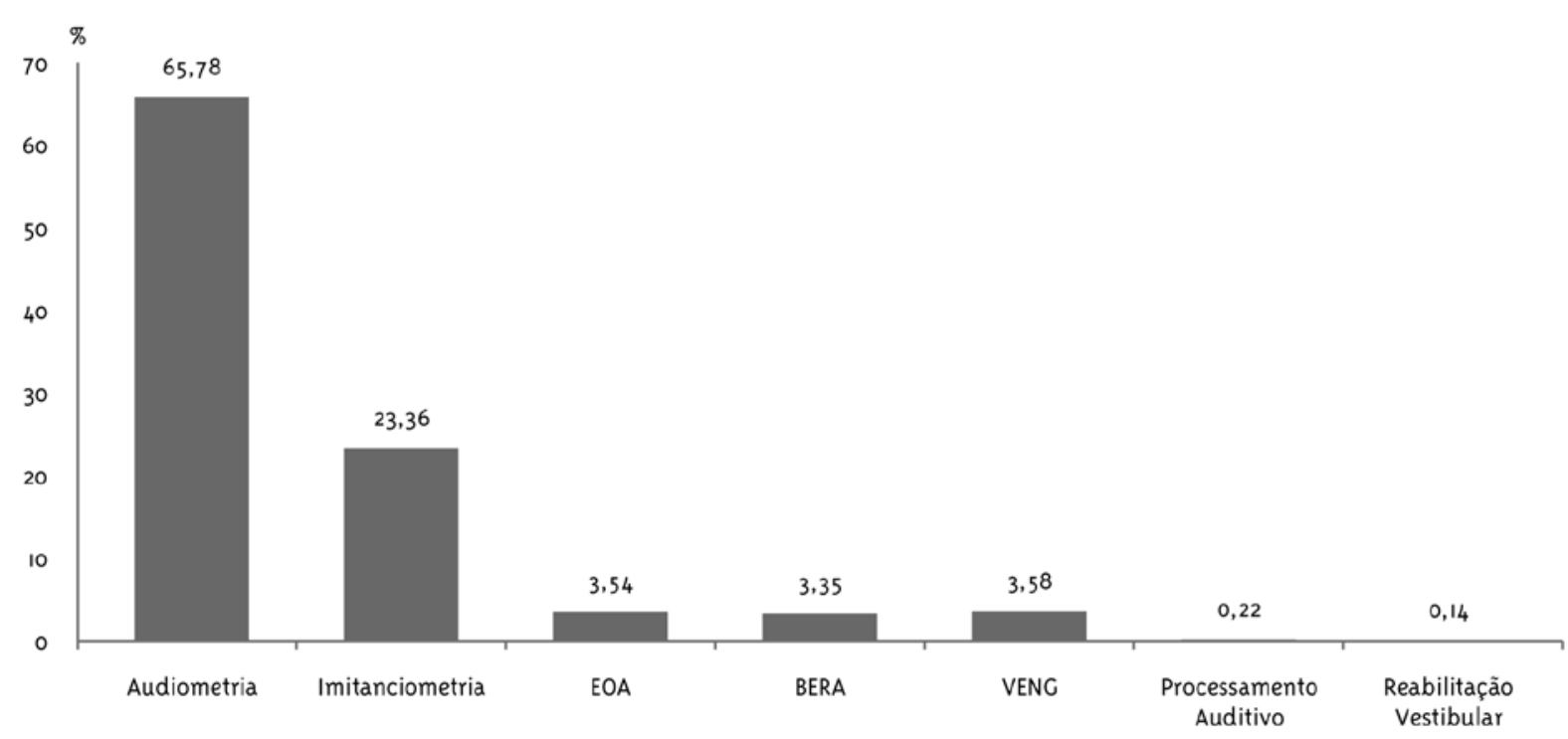


Os dois últimos, apesar de terem se tornado bastante conhecidos nos últimos anos, ainda são solicitados, geralmente, por neurologistas ou otorrinolaringologistas, que possuem maior conhecimento sobre a necessidade de sua realização para a investigação diagnóstica de seus pacientes. 0 exame de emissões otoacústicas (EOA) possui grande aplicação clínica na triagem auditiva neonatal, sendo capaz de testar a capacidade de funcionamento da cóclea, órgão sensorial da audição, de modo objetivo, simples, rápido e não invasivo. Desde 1993, nos EUA, o National Institute of Health (NIH) recomenda o teste para todos os bebês até os três meses de vida, visando ao diagnóstico precoce da perda auditiva (Lopes Filho e Carlos, 2005). Em Fortaleza, a Lei Municipal $n^{0}$ 9.162, de 22/2/2007, regulamenta a realização gratuita do exame em todas as maternidades do município, determinando a necessidade do fonoaudiólogo também nessas instituições.

Os procedimentos referentes à avaliação do processamento auditivo, o,22\% (48), e reabilitação vestibular, o,14\% (30), apareceram com valores sem significância.

\section{Origem dos encaminhamentos para exames au- diológicos}

Na figura 2, é possível verificar as origens dos encaminhamentos dos pacientes para a realização de exames audiológicos. 0 médico otorrinolaringologista foi apontado como o principal especialista responsável pelo encaminhamento aos exames, com $56,39 \%$ (450) dos 798 prontuários analisados. 0 alto índice de encaminhamentos otorrinolaringológicos justifica-se pelo julgamento dos pacientes de ser esse o modo possível para o registro formal de suas queixas e solicitação de avaliação audiológica. Além disso, a otorrinolaringologia e a fonoaudiologia são duas áreas intrinsecamente relacionadas, havendo, no serviço de fonoaudiologia do NAMI, atendimento otorrinolaringológico disponível.

Tornou-se evidente a diversidade de profissionais de outras áreas e especialidades que passaram a reconhecer a importância e a necessidade de realizar esses exames, apesar de não haver significância em termos percentuais.

\section{Figura 2 - Origem do encaminhamento no setor de audiologia do NAMI, 2006-2007}

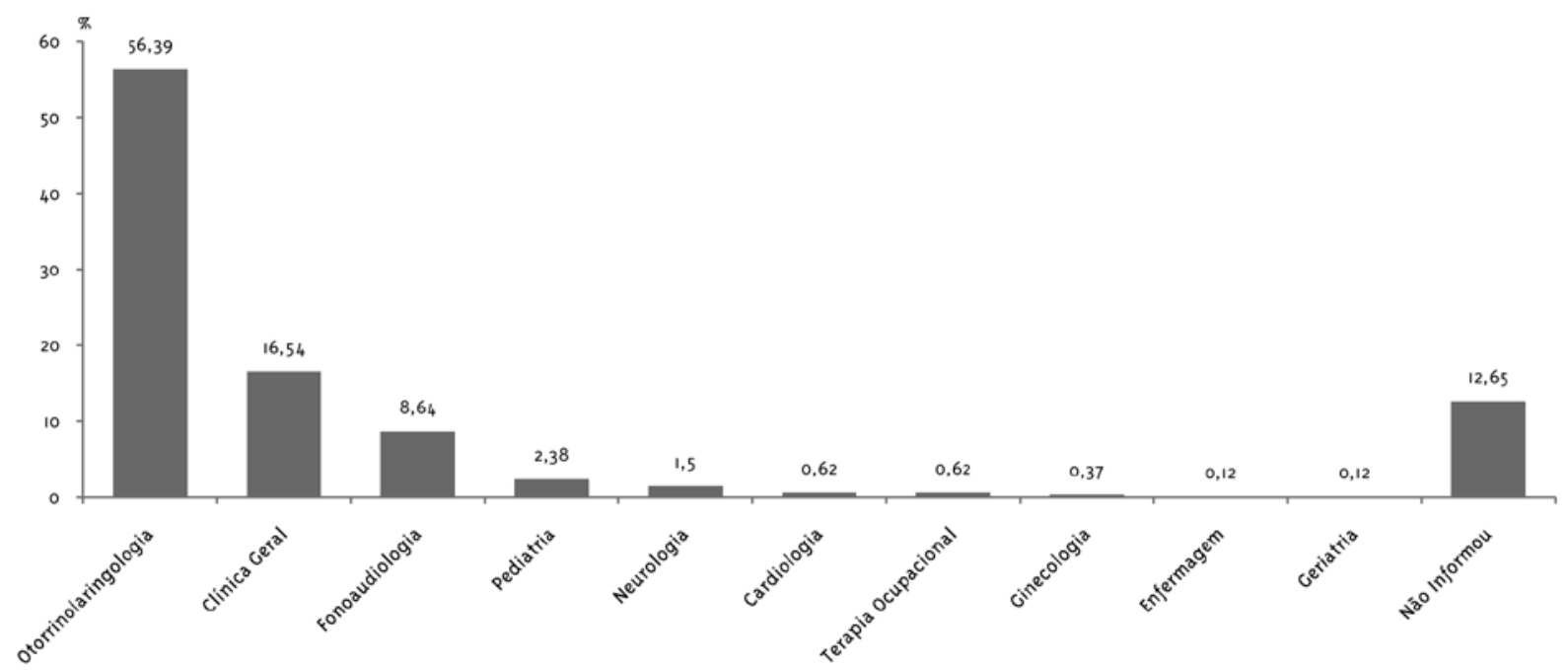


O item "fonoaudiologia”, ainda na figura 2, refere-se aos encaminhamentos realizados pelos professores da disciplina de Fonoaudiologia em Saúde Coletiva durante as visitas domiciliares na Comunidade e, ainda, por fonoaudiólogos de outros serviços, correspondendo a 8,64\% (69). 0 item "clínica geral" faz referência aos encaminhamentos originados das unidades básicas de saúde, pelos médicos da ESF, que apresentou um percentual de $16,54 \%$ (132), aparecendo em segundo lugar. Esse fato demonstra a necessidade do fonoaudiólogo na unidade básica de saúde, a fim de favorecer o princípio da resolutividade de maneira mais efetiva. Observa-se que o item "não informado" obteve percentual significativo, com $12,65 \%$, considerando que 101 prontuários avaliados não informavam a origem do encaminhamento.

Não foi possível verificar o nível de escolaridade, bem como delimitar o perfil socioeconômico da população atendida no setor de audiologia do NAMI, devido ao fato de essas informações não estarem contidas em nenhum dos formulários utilizados durante o comparecimento do paciente para a realização dos exames.

\section{Perfil da demanda no serviço de fonoterapia}

Quanto à análise dos prontuários de atendimento no setor de fonoterapia, foram estudados os as- pectos referentes a gênero, faixa etária, área de atendimento, nível de escolaridade, renda familiar, tempo de espera para o início do atendimento, tempo de permanência em terapia e conclusão do tratamento. Esse estudo foi realizado a partir dos 346 prontuários referentes aos anos de 2006 e 2007, constatando-se o predomínio do gênero masculino, com 52,60\% (182), em relação ao feminino, que aparece com 47,39\% (164). Foi ressaltada a faixa etária entre o e 10 anos, correspondendo a 58,09\% (201). As idades compreendidas entre 11 e 20 anos, $16,76 \%$ (58); 21 e 40 anos, $16,18 \%$ (56); 41 e 65 anos, $6,64 \%$ (23) e maiores de 65 anos 2,89\% (10), aparecerem com menor percentual.

\section{Áreas de atendimento em fonoterapia}

Na figura 3, são apresentadas as informações referentes à área de atendimento predominante, com prevalência para as alterações de linguagem oral e/ou escrita, com 36,99\% (128), seguida por distúrbios de motricidade orofacial, com 28,03\% (97) e alteração dos aspectos vocais, que correspondem a 27,45\% (95). 0 ambulatório de espasticidade aparece com 6,64\% (23) dos prontuários estudados. 0 item "sem alterações", o,28\% (1), diz respeito ao caso do paciente que compareceu ao serviço, sendo avaliado, entretanto, não havendo constatação da queixa ou de qualquer alteração fonoaudiológica.

\section{Figura 3 - Área de atendimento em fonoterapia no NAMI, 2006-2007}

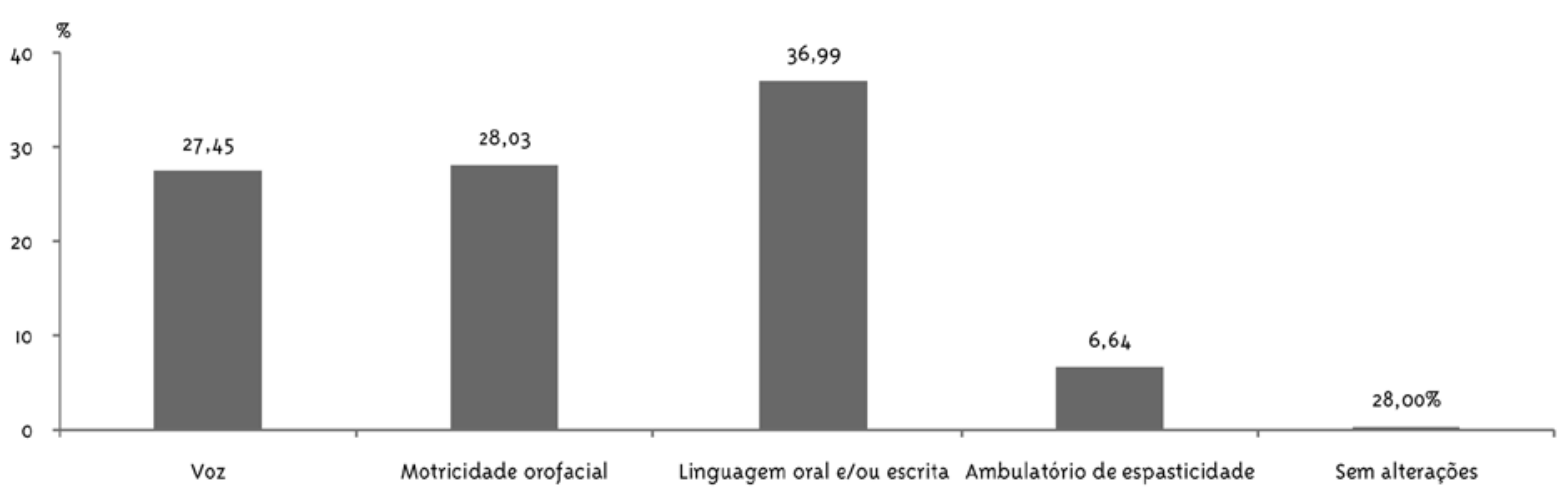




\section{Caracterização do perfil da demanda em fonote- rapia}

O predomínio do gênero masculino em relação ao feminino caracteriza a confirmação de achados observados em estudos anteriores (César e Maksud, 2007; Gonçalves e col., 2000; Hage e Faiadha, 2005; Choudury e Benasich, 2003), sendo pertinente uma pesquisa específica para a investigação dessas informações, já que a prevalência de alterações no gênero masculino é apontada em vários estudos sem explicações comprovadas. $\mathrm{O}$ argumento significativo para esse dado refere-se ao fato de que o cérebro masculino apresenta uma maturação mais lenta que o feminino (César e Maksud, 2007; Hage e Faiadh, 2005), associada à questão genética, apontada como determinante na prevalência de distúrbio específico de linguagem em meninos.

Outros estudos demonstraram prevalência da população entre 5 e 10 anos, seguida pela de o a 5 anos (César e Maksud, 2007; Gonçalves e col., 2000; Hage e Faiad, 2005; Choudury e Benasich, 2003). A demanda maior nessas faixas etárias reflete um dado importante e se deve ao fato de que a criança está iniciando novas interações sociais, com participação ativa na escola, onde os aspectos linguísticos são mais cobrados. Esse momento exige novas habilidades, de modo que tornam mais perceptíveis as alterações de fala e linguagem.

$\mathrm{Na}$ maioria dos casos, a criança necessita de intervenção fonoaudiológica direta, e as faixas etárias citadas anteriormente representam o período crítico para essa atuação. Assim, estando esse profissional presente nas unidades básicas de saúde, as chances de melhoria do desenvolvimento linguístico de crianças seriam maiores, considerando o acesso direto da população ao serviço de fonoaudiologia, evitando, assim, prejuízos associados, como mau desempenho na escola e problemas de interação social, contribuindo para a elevação da qualidade de vida. As alterações de linguagem oral e/ou escrita seguida de queixas relacionadas à motricidade orofacial também foram encontradas em outros estudos (Lipay e Almeida, 2007; César e Maksud, 2007; Marin e col., 2003; Wertzner, 1997).

Durante o levantamento do nível de escolaridade dos pacientes identificados nos prontuários de fonoterapia, procedeu-se da seguinte maneira: para os menores de 18 anos, foram verificadas as informações referentes ao grau de instrução dos pais, utilizando-se como critério de escolha entre o pai e a mãe, o que apresentasse o maior grau, a fim de caracterizar o nível sociolinguístico da criança. Para os pacientes maiores de 18 anos, essa informação foi adquirida a partir da verificação do nível de escolaridade do paciente, estando esses dados assim distribuídos: nível médio completo, 28,32\% (98); não informado, 25,14\% (87); nível superior completo, $12,42 \%$ (43); nível médio incompleto, 8,38\% (29); superior incompleto, 7,22\% (25); fundamental completo, 6,06\% (21). É importante conhecer o nível de escolaridade do paciente e das pessoas inseridas em seu meio social, considerando que os aspectos ambientais exercem influência para a formação pessoal, e o sucesso do processo terapêutico também se deve à participação da família.

Durante a coleta, foi identificada, em muitos prontuários, a ausência de dados importantes para caracterizar a demanda da população atendida, tais como renda familiar e nível de escolaridade, que constituíram o item "não informado". No setor de audiologia, essas informações não foram computadas, pois não constam das fichas, sugerindo-se aqui sua inclusão. No setor de fonoterapia, esses dados constam das fichas, porém, devido à desinformação dos pacientes e/ou responsáveis, ou à desvalorização das informações por parte dos alunos responsáveis pelo atendimento, não são devidamente informados.

No que se refere à renda, houve prevalência das famílias que recebem entre um e três salários mínimos, com 33,23\% (115), seguidas daquelas que recebem menos de um salário mínimo mensal, que correspondem a $14,73 \%(51)$. A renda mensal acima de três salários mínimos apareceu com 12,42\% (43). O item "não informado" constou em 39,59\% (137) dos prontuários. Conhecer as condições socioeconômicas da população atendida revela um dado significativo para o prognóstico do processo terapêutico.

Apesar de ser um serviço público, é possível perceber muitos casos no NAMI nos quais o paciente deixa de comparecer à terapia por não possuir o dinheiro da condução, tornando evidente a importância da distribuição do serviço fonoaudiológico em outras regiões de Fortaleza. Estando o fonoaudiólogo presente em cada unidade básica de saúde, o 
acesso dessas pessoas ao serviço seria facilitado.

\section{Conclusão do tratamento em fonoterapia}

Na dinâmica de funcionamento do setor de fonoterapia do NAMI, o paciente tem o atendimento cancelado e perde a vaga no serviço após duas faltas consecutivas sem justificativa plausível. Esse critério foi definido com base na necessidade de vagas para atendimento, considerando a procura pelo serviço. A quantidade de cancelamentos se configurou em maior número, aparecendo em 65,89\% (228) dos casos. Esse registro pode estar relacionado ao item "renda familiar", descrito anteriormente. A alta ocorreu em 27,74\% (96) dos casos. Houve, ainda, o registro de casos de desistências do atendimento, com 6,64\% (23) (Figura 4).

\section{Figura 4 - Conclusão do tratamento no setor de fono- terapia no NAMI, 2006-2007}

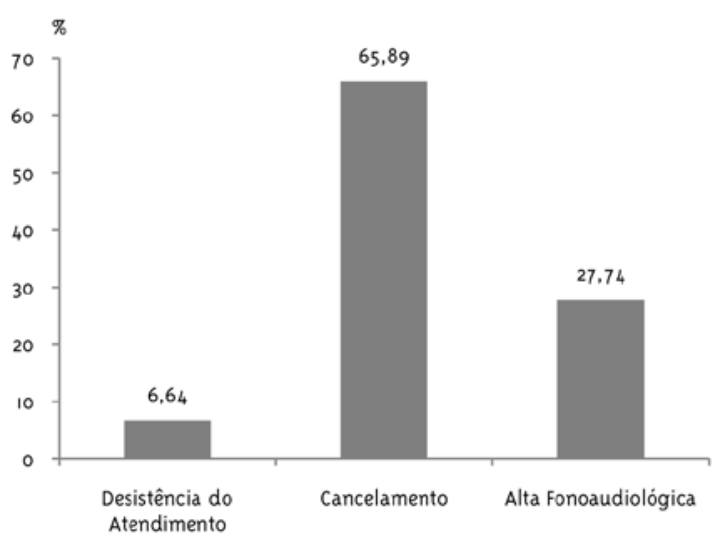

Faz-se necessária a realização de outros estudos nessa área, que possam investigar os fatores que levam ao elevado número de cancelamentos, de modo a contribuir para a melhoria do serviço oferecido.

\section{Tempo de espera para atendimento fonoterápico}

O intervalo com maior prevalência foi de o a 3 meses, com 54,62\% (189); em seguida, apareceram os pacientes que esperaram entre 4 e 6 meses, com 22,54\% (78) e 7 a 9 meses, com 10,69\% (37). Em menor proporção, apareceram os pacientes que aguardaram de 10 a 12 meses, com 6,35\% (22) e tempo superior a 12 meses, com 5,78\% (20). Para a aquisição dessa informação, foi calculado o intervalo entre a data registrada no relatório de avaliação e a data presente no primeiro relatório de atendimento fonoterápico do paciente, logo após a avaliação, considerando que é rotina da instituição fornecer relatório semestral contendo a descrição da situação do paciente (Figura 5).

\section{Figura 5 - Tempo de espera para atendimento no setor} de fonoterapia no NAMI, 2006-2007

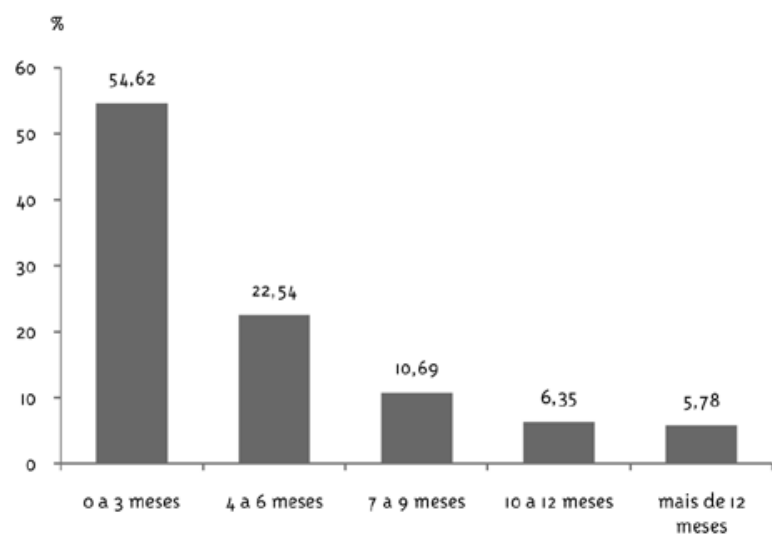

Tempo de permanência em fonoterapia

Verificou-se o intervalo de tempo entre a data do primeiro e do último relatório de atendimento do paciente. 0 período de o a 6 meses foi o de maior prevalência, com 34,39\% (119); seguido do período de 7 a 12 meses, com 24,56\% (85); e 25 a 30 meses, com 19,65\% (68). Os períodos de 13 a 18 meses, e 19 a 24 meses foram os que obtiveram menor prevalência, com $11,56 \%$ (40) e 9,82\% (34), respectivamente (Figura 6).

\section{Figura 6 - Tempo de permanência em fonoterapia no NAMI, 2006-2007}

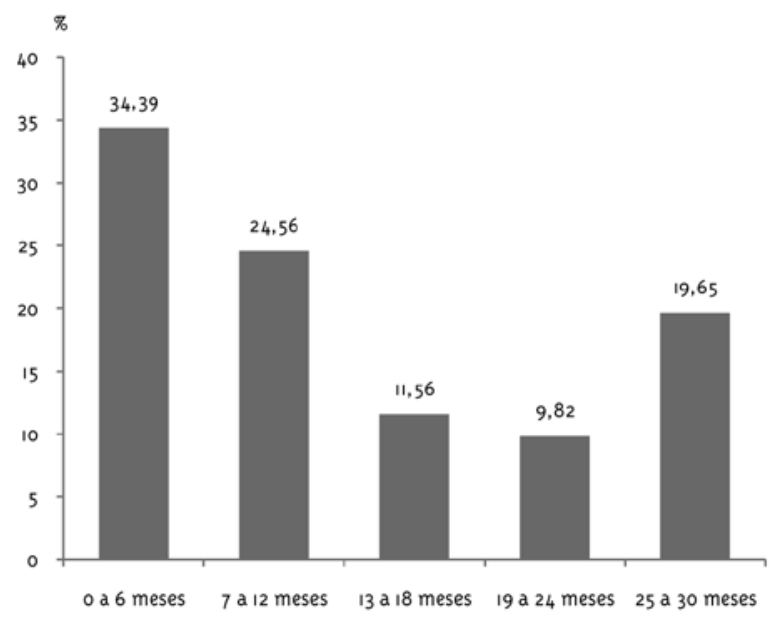


Outra evidência significativa está relacionada ao trabalho com os grupos de orientação, que faz parte de uma proposta integradora e está presente em todas as áreas de atendimento fonoaudiológico do NAMI, correspondendo a 37,33\% (193) das modalidades de atendimento, no ano de 2007. A terapia individual apresentou maior prevalência, com $62,66 \%$ (324). Foi observado, ainda, um pequeno crescimento do número de atendimentos em grupo, a partir da comparação dos anos de 2006 e 2007 (Figura 7). Esse fato demonstra o sucesso dessa modalidade de atendimento. Outros estudos mostram a importância da atuação em grupos, os quais possibilitam o envolvimento dos sujeitos em atividades de sensibilização, favorecendo a reflexão e mudança, a educação, a socialização, a terapia e a comunicação de modo integrado. Essa modalidade se mostra favorável ao processo de ensino, pesquisa e extensão em fonoaudiologia (Penteado, 2003).

A partir desse levantamento, tornou-se clara a necessidade da inserção do fonoaudiólogo na rede municipal de saúde do município de Fortaleza. 0 atendimento no serviço de fonoaudiologia do NAMI, em sua maioria, é realizado pelos alunos do curso de graduação, sob supervisão dos professores. Durante os meses de dezembro, janeiro, junho e julho, que
Figura 7 - Comparativo entre atendimento individual e em grupo no NAMI, 2006-2007

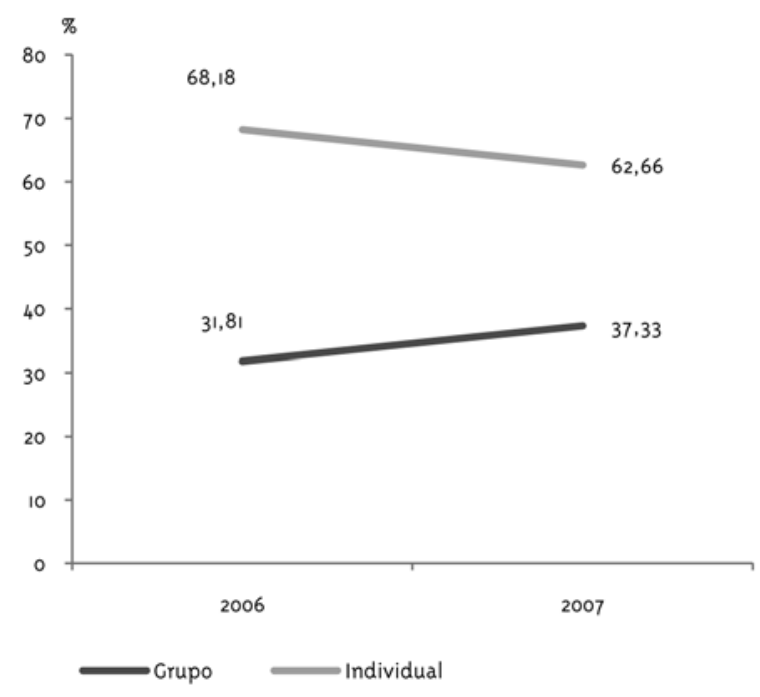

\section{Figura 8 - Número de atendimentos nos setores de audiologia e fonoterapia no NAMI, 2006-2007}

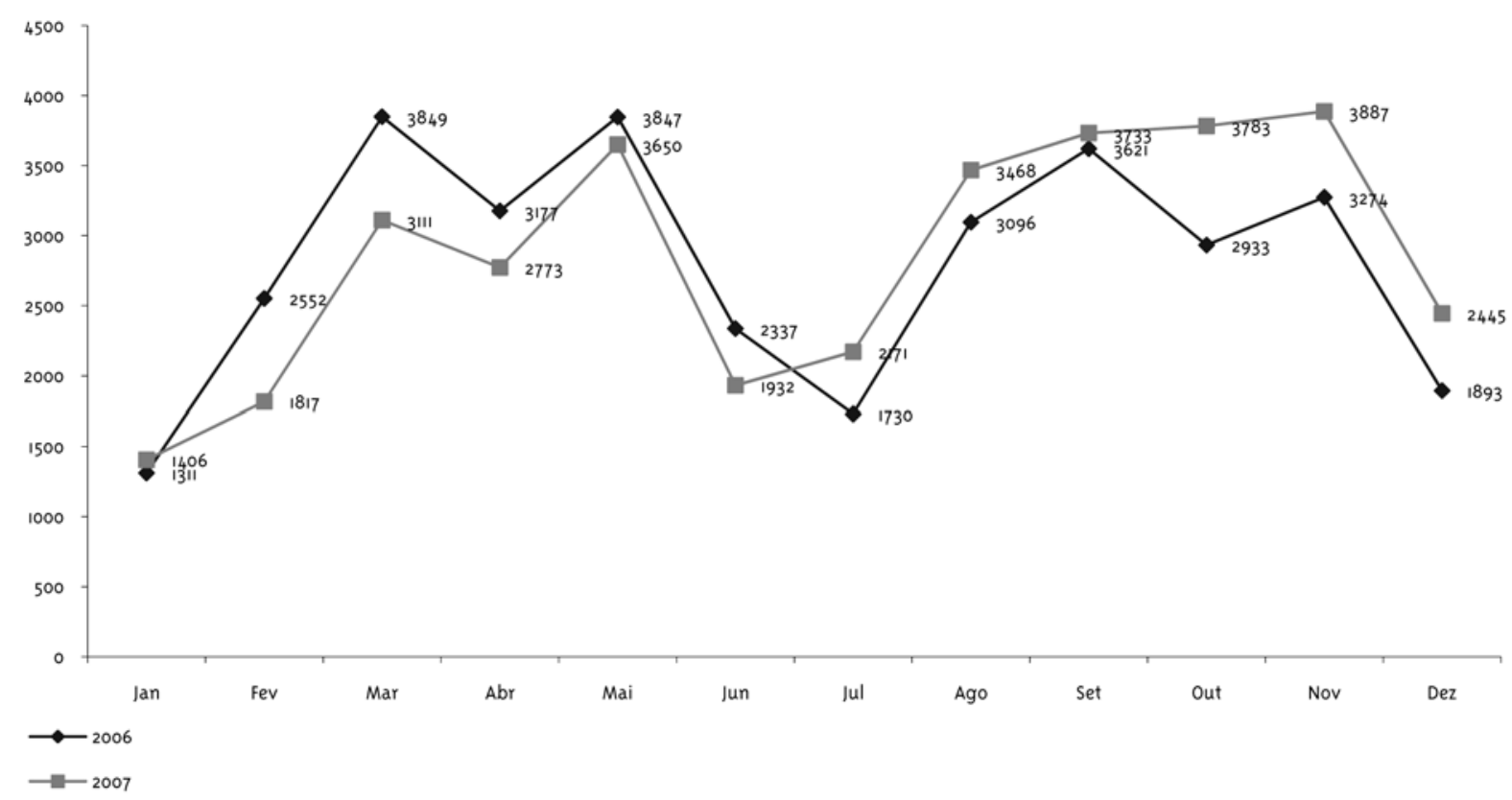


O número de atendimentos, a cada semestre, sofreu influência da quantidade de alunos por turma, havendo necessidade de suspensão e remanejamento de atendimentos quando da ocorrência da redução do número de turmas práticas. Essa interrupção repercutiu na evolução do processo terapêutico, contribuindo para o aumento da fila de espera e do período da terapia, e da redução no índice de exames audiológicos realizados. Além disso, ao início de cada semestre, um novo aluno assumia a terapia dos pacientes que seguiam em atendimento, sendo necessário um período de adaptação entre terapeuta e paciente, necessária à evolução do processo terapêutico, o que aumentou o curso da terapia.

Com a presença do fonoaudiólogo nas unidades básicas, além da promoção da saúde e atendimento em nível primário, seria possível detectar precocemente as alterações e haveria continuidade do trabalho de modo mais efetivo, otimizando o processo de intervenção e evitando a ocorrência de alterações associadas.

\section{Conclusão}

O serviço de fonoaudiologia do NAMI-Unifor disponibiliza apenas vinte vagas mensais pelo sistema de agendamento on-line, destinadas à população do município de Fortaleza, que é encaminhada através das unidades básicas de saúde. Essas vagas incluem todas as áreas de atendimento fonoterápico, bem como triagem, avaliação, participação em grupos terapêuticos e terapia individual, além da realização de exames, sendo esse um número aquém das necessidades apresentadas pela população.

Este estudo mostra a importância da contratação de mais profissionais para o serviço municipal de saúde, no sentido de minimizar os impactos gerados pela flutuação do número de alunos responsáveis pelo atendimento nos diversos períodos do ano, já que, como clínica-escola, o NAMI preza pela responsabilidade, qualidade e continuidade das atividades prestadas à população.

Para a realização de um trabalho efetivo em qualquer área de atuação, é fundamental o conhecimento do serviço no qual se atua para delimitar seus limites e possibilidades. Este estudo demonstra que o serviço de fonoaudiologia do Núcleo de Atenção Médica
Integrada (NAMI), da Universidade de Fortaleza (Unifor), vem contribuindo de maneira significativa para o reconhecimento e a ampliação da profissão e a melhoria da saúde da comunicação humana da população desse município e regiões vizinhas. Entretanto, faz-se necessária a inserção do fonoaudiólogo na rede municipal de saúde de Fortaleza para tornar mais acessível os serviços oferecidos no que concerne à saúde da comunicação humana, sobretudo, no nível de atenção primária, concretizando o conceito de saúde e efetivando, na prática, os princípios do Sistema Único de Saúde.

\section{Referências}

BASÍLIO, C. S. et al. Fonoaudiologia e saúde coletiva. Revista Paulista de Pediatria, São Paulo, v. 18, n. 4, p. 179-18o, dez. 2000.

BEFI, D. A inserção da fonoaudiologia na atenção primaria à saúde. In:_BEFI, D.(org.). Fonoaudiologia na atenção primaria à saúde. São Paulo: Lovise, 1997. p. 15-36.

BLEICHER, L. Saúde para todos, já! Fortaleza: Expressão Gráfica, 2004.

BRASIL. Constituição Federal (1988). Constituição da República Federativa do Brasil. Brasília, DF: Senado Federal, 1988.

BRASIL. Ministério da Saúde. O SUS e os cursos de graduação na área de saúde: aprender SUS. Brasília, DF, 2004. Disponível em: <http://portal. saude.gov.br/portal/aplicacoes/>. Acesso em: 13 abr. 2008.

BRASIL. Ministério da Saúde. Portaria $N^{o} 154$, de 24 de Janeiro de 20o8. Criação dos Núcleos de Apoio à Saúde da Família. Brasília, DF, 2008. Disponível em: <http://bvsms.saude.gov.br/bvs/ saudelegis/gm/2008/prto154_24_01_2008.html>. Acesso em: 15 abr. 2008.

CECCIM, R. B.; FEUERWERKER, L. C. M. Mudança na graduação das profissões de saúde sob o eixo da integralidade. Cadernos de Saúde Pública, Rio de Janeiro, v. 20, p. 1400-1410, set./out. 2004. Disponível em: <http://www.scielo.br/pdf/csp/ v2on5/36.pdf>Acesso em: 13 abr. 2008. 
CECCIM, R. B.; FEUERWERKER, L. C. M. O quadrilátero da formação para a área da saúde: ensino, gestão, atenção e controle social. PHYSIS: Revista de Saúde Coletiva, Rio de Janeiro, v. 14, n. 1, p. 41-65, 2004.

CÉSAR, A. M.; MAKSUD, S. S. Caracterização da demanda de fonoaudiologia no serviço público municipal de Ribeirão das Neves - MG. Revista CEFAC, São Paulo, v. 9, n. 1, p. 133-138, jan./mar. 2007.

CHOUDHURY, N.; BENASICH, A. A. A family aggregation study: the influence of family history and other risk factors on language development. Journal of Speech, Language, and Hearing Research, New Jersey, v. 46, n. 2, p. 261-272, apr. 2003.

CHUN, R. Y. S. Promoção da saúde e as práticas em fonoaudiologia. In: FERREIRA, L. P.; BEFI, D. M.; LIMONGI, S. C. O. (orgs). Tratado de fonoaudiologia. São Paulo: Roca, 2004. p. 538-544.

FORTALEZA (Município). PREFEITURA MUNICIPAL DE FORTALEZA. Lei n. 9.162, de 22 de fevereiro de 2007. Regulamenta a realização gratuita do exame de emissões otoacústicas nas maternidades do município. Disponível em: <http://www.pgj.ce.gov.br/asscomv2>. Acesso em: 18 set. 2008.

GONÇALVES, C. G. O. et al. Demanda pelos serviços de fonoaudiologia no município de Piracicaba: estudo comparativo entre a clínicaescola e o atendimento na Prefeitura Municipal. Pró-Fono, São Paulo, v. 12, n. 2, p. 61-66, set. 2000.

GONÇALVES, M. S. et al. Fonoaudiologia e saúde coletiva: prioridades detectadas pelos usuários de unidades básicas de saúde. Revista Fonoaudiologia Brasil, Brasília, DF, v. 3, n. 2, p. 1-3, abr./jun. 2005 .

GOULART, B. N. G. A fonoaudiologia e suas inserções no Sistema Único de Saúde: análise prospectiva. Revista Fonoaudiologia Brasil, Brasília, DF, p. 29-34, dez. 2003.
HAGE, S. R. V.; FAIAD, L. N. V. Perfil de pacientes com alteração de linguagem atendidos na clínica de diagnóstico dos distúrbios da comunicação. Revista CEFAC, São Paulo, v. 7, n. 4, p. 433-44o, out./dez. 2005.

LIPAY, M. S.; ALMEIDA, E. C. A fonoaudiologia e sua inserção na saúde pública. Revista Ciências Médicas, Campinas, SP, v.16, n. 1, p. 31-41, jan./fev. 2007.

LOPES FILHO, O.; CARLOS, R. C. Emissões otoacústicas. In: LOPES FILHO, O. Tratado de fonoaudiologia. Ribeirão Preto, SP: Tecmedd, 2005. p. 207-222.

MARIN, C. R. et al. Promoção da saúde em fonoaudiologia: ações coletivas em equipamentos de saúde e de educação. Revista da Sociedade Brasileira de Fonoaudiologia, São Paulo, n. 1, p. 35-41, jun. 2003.

MENDES, V. L. F. Acesso à saúde em fonoaudiologia. In: BEFI, D. (org.). Fonoaudiologia na atenção primária à saúde. São Paulo: Lovise, 1997. p. 43-56.

MENDES, V. L. F. Fonoaudiologia e saúde coletiva: perspectivas de atuação nos serviços públicos de saúde. Distúrbios da Comunicação, São Paulo, v. 10, n. 2, p. 213-24, jun. 1999.

PAOLETTI, M. R. M. Participação da fonoaudiologia na atenção primária à saúde, caracterização da população atendida pelo serviço de fonoaudiologia das unidades básicas de saúde do município de Embu, São Paulo (1986/87). 1992. 173 f. Dissertação (Mestrado)-Universidade Federal de São Paulo, São Paulo, 1992.

PENTEADO, R. Z. Grupo ou agrupamento? Estudo da constituição de um grupo em fonoaudiologia. In: MARCHESAN, I.; ZORZI, J. (orgs.). Tópicos em fonoaudiologia. Rio de Janeiro: Revinter, 2003. p. 33-59.

PENTEADO, R. Z.; SERVILHA, E. A. M. Fonoaudiologia em saúde pública/coletiva: compreendendo prevenção e o paradigma da promoção da saúde. Distúrbios da Comunicação, São Paulo, v. 16, n. 1, p. 107-116, abr. 2004. 
PORTMANN, M.; PORTMANN, C. Tratado de

audiometria clínica. 6. ed. São Paulo: Roca, 1993.

PRETTO, S. M.; FRANCO, F. Visitas domiciliares

com estratégia de educação em saúde: relato de uma experiência. Ação Coletiva, Porto Alegre, v. 2, n. 3, p. 33-44, jul./set. 1999.

SILVA, R. M.; ARAÚJO, M. A. L. Promoção da saúde no contexto interdisciplinar. Revista Brasileira em Promoção da Saúde, Fortaleza, v. 20, n. 3, p. 141-

142, 2007.

VERAS, R. P; MATTOS, L. C. Audiologia do envelhecimento: revisão da literatura e perspectivas atuais. Revista Brasileira de Otorrinolaringologia, São Paulo, v. 73, n. 1, p.128134, jan./fev. 2007.

WERTZNER, H. F. Ambulatórios de fonoaudiologia em unidades básicas de saúde. In: BEFI, D. (Org.). Fonoaudiologia na atenção primária à saúde. São Paulo: Lovise, 1997. p. 161-176. 